\title{
Viscoelasticity of Carp Actomyosin Solution during Heating
}

\author{
Teruo Nakayama,* Masanori Tsunekawa,* Satoshi Kanoh,* \\ and Eiji Niwa* \\ (Received March 17, 1988)
}

\begin{abstract}
The steady and dynamic shear rate behaviors of carp actomyosin solution were measured during incubation at $40^{\circ} \mathrm{C}$. The maximum steady shear viscosity was observed around $35 \mathrm{~min}$, while the maximum dynamic rigidity was observed around $2 \mathrm{~h}$. The hysteresis loop area increased for $2 \mathrm{~h}$, and thereafter it remained almost unchanged till $6 \mathrm{~h}$ where the dynamic rigidity decreased. Therefore we deduce that during the incubation, the network structure would develop also in the carp actomyosin solution as in the hen or rabbit myosin solution, but the network structure of carp actomyosin solution would be so weak as to be broken down even during the repeated measurements of dynamic behaviors.

The actomyosin solution at $\mathrm{pH} 5.8$ exhibited the lowest viscoelasticity. The solution at $\mathrm{pH}$ 6.8 exhibited the highest dynamic rigidity. The solution at $\mathrm{pH} 7.8$ exhibited the highest stress in the shear rate scan measurement. These results were explained by the fact that the actomyosin solution at $\mathrm{pH} 5.8$ existed near the isoelectric point, and the network structure at $\mathrm{pH} 6.8$ would be different from the network structure at $\mathrm{pH} 8.8$.

In the temperature scanning experiment the dynamic rigidity began to increase around $40^{\circ} \mathrm{C}$ the maximum was observed around $61^{\circ} \mathrm{C}$, The peak value around $61^{\circ} \mathrm{C}$ was high at $\mathrm{pH} 5.8$ and pH 6.8, and low at pH 7.8.
\end{abstract}

When carp myosin solutions $\left(9.1 \mathrm{mg} / \mathrm{m} l, 35^{\circ} \mathrm{C}^{\mathrm{b})}\right)$ and $6.3 \mathrm{mg} / \mathrm{m} l, 40^{\circ} \mathrm{C}^{2)}$ ) were incubated, the viscosity once increased and then decreased. However when hen or rabbit myosin solutions $(9.1 \mathrm{mg} /$ $\mathrm{mg}, 35^{\circ} \mathrm{C}^{1)}$ and $6.3 \mathrm{mg} / \mathrm{ml}, 40^{\circ} \mathrm{C}^{2)}$ ) were incubated, the viscosity increased and leveled off around the maximum value. Then we tried to investigate also carp actomysoin in this viewpoint. When carp actomyosin solutions $\left(12.5 \mathrm{mg} / \mathrm{ml}, 40^{\circ} \mathrm{C}\right)^{33}$ were incubated, the viscosity once increased and then decreased.

These differences in viscosity change between carp protein and domestic animal protein might be revealed by the breakdown of the network structure of carp proteins during the viscosity measurement which brought about large deformation. Inherent properies of carp myofibrillar proteins might be similar to those of hen or rabbit myofibrillar proteins if viscoelasticity is measured within small deformation range. Therefore the repeated measurements of both the steady shear viscosity and the dynamic visoelasticity of carp actomyosin solution were carried out during the course of incubation at $40^{\circ} \mathrm{C}$ to investigate the formation and breakdown of network structure in it.
In addition, we elevated the temperature of carp actomyosin solutions up to $80^{\circ} \mathrm{C}$ and monitored their viscoelastic properties during this heating process.

\section{Materials and Methods}

\section{Preparation of Actomyosin Solutions}

Carp actomyosin solution was prepared by Takashi's procedure, ${ }^{4}$ except that $\mathrm{NaCl}$ was used instead of $\mathrm{KCl}^{3,5}$ The protein concentration was determined by biuret method with the calibration by means of the micro-Kjeldahl method.

\section{Incubation of Actomyosin Solutions}

Actomyosin concentration was adjusted to $19 \mathrm{mg} / \mathrm{ml}$ by diluting the stock solution. This concentration was high enough to observe the thixotropic properties of actomyosin solution. To measure the viscoelasticity change, actomyosin solution was incubated at $40^{\circ} \mathrm{C}$ in the gap between coaxial cylinders of a Toyo Seiki rheometer (details on this device will be written in the next section). Coaxial cylinders were made up of a cup and a bob. The cup was at first kept at $5^{\circ} \mathrm{C}$,

* Faculty of Fisheries, Mie University, Tsu 514, Japan (中山照雄, 常川正則, 加納 哲, 丹羽栄二：三重 大学水産学部). 
and the cold actomyosin solution $\left(5^{\circ} \mathrm{C}\right)$ was poured in it. Then the bob was set to dip into the solution. From this moment, the temperature was elevated up to $40^{\circ} \mathrm{C}$ using Fix Mode of programmable temperature regulator (Rika Kogyo Co., Ltd., Tokyo). It took 6 min to arrive at the set temperature of $40^{\circ} \mathrm{C}$. The moment when the temperature of the actomyosin solution just became $40^{\circ} \mathrm{C}$, was taken as zero incubation time. The temperature change was monitored by the thermocouple, and was indicated on the LED display of temperature regulator. The dynamic and steady shear rate responses of actomyosin solution were measured at $40^{\circ} \mathrm{C}$, and the shear rate scan behavior was measured at $5^{\circ} \mathrm{C}$.

Measurements of Dynamic, Steady Shear Rate, Shear Rate Scan, and Temperature Scan Behaviors

Dynamic, steady shear rate, shear rate scan, and temperature scan behaviors were measured with a Toyo Seiki rheometer (Rheologiraph Liquid, Toyo Seiki Manufacturing Co., Ltd., Tokyo) fitted with coaxial cylinders $(\mathrm{Ri} / \mathrm{Ro}=$ 0.90 ). The inner cylinder (bob) was suspended by a torsion wire which was centrered by means of air bearing system. The outer cylinder (cup) was vibrated (frequency: 1 or 3 cycle/s, strain amplitude: 0.18 ) or rotated (shear rate: 6,12 , or $48 \mathrm{~s}^{-1}$ in the steady shear rate measurement, and $1 \mathrm{~s}^{-1} \rightarrow 50 \mathrm{~s}^{-1} \rightarrow 1 \mathrm{~s}^{-1}$ in the shear rate scan measurement). The sample temperature was controlled by a thermomodule jacket surrounding the measuring head.

Just after a constant vibration or a constant shear rate was applied to the sample for $1 \mathrm{~min}$ at $40^{\circ} \mathrm{C}$, the resultant torsion signal was incorporated in the operation system, from which the dynamic rigidity/the dynamic loss, or the shear stress was derived to the chart, respectively. This measurement of dynamic or steady shear rate behavior was carried out at intervals of every ten minutes during the incubation at $40^{\circ} \mathrm{C}$.

The degrees of hysteresis were measured with increasing and decreasing shear rate determinations by the use of Scan Mode at $5^{\circ} \mathrm{C}$, after the actomyosin solution was incubated for a given time at $40^{\circ} \mathrm{C}$. The shear rate was increased from $1 \mathrm{~s}^{-1}$ to $50 \mathrm{~s}^{-1}$, and then decreased to $1 \mathrm{~s}^{-1}$. It was linearly changed with time. The shear rate vs. shear streess curve was automatically drawn on the chart. One scan required $100 \mathrm{~s}$.

The ecffect of temperature on the viscoelastic properties of actomyosin solution was investigated by the use of Program Mode of temperature regulator. The temperature of actomysoin solution was elevated up to $80^{\circ} \mathrm{C}$ to keep the heating rate at $1^{\circ} \mathrm{C} \mathrm{min}^{-1}$.

Throughout all the present experiments, liquid paraffin was layered on the top of the actomyosin solution in the gap between coaxial cylinders to prevent the moisture evaporation.

\section{Results and Discussion}

\section{Breakdown of Network Structure during Repeated} Measurements of Steady and Dynamic Shear Rate Behaviors

The steady shear viscosity $\eta$ of carp actomyosin solution $(19 \mathrm{mg} / \mathrm{m} l)$ increased till the incubation time of about $35 \mathrm{~min}$ and then decreased, during the incubation at $40^{\circ} \mathrm{C}$ (Fig. 1). The dynamic rigidity $\mathrm{G}^{\prime}$ of carp actomyosin solution increased till the incubation time of about $2 \mathrm{~h}$ and then decreased during the incubation (Fig. 2).

The steady shear viscosity $\eta$ was measured at the shear rates of 6,12 , and $48 \mathrm{~s}^{-1}$, The viscosity value became smaller at any incubation time when the higher shear rate was used. But the tendency of viscosity change during the incubation was similar irrespective of the shear rate used. In Fig. 1, the steady shear viscosity measured at $12 \mathrm{~s}^{-1}$, was shown representatively. The dynamic rigidity $G^{\prime}$ was measured at the frequencies of 1 and 3 cycle $\mathrm{s}^{-1}$. The dynamic rigidity value became larger at any incubation time when the highter frequency was used. But the tendency

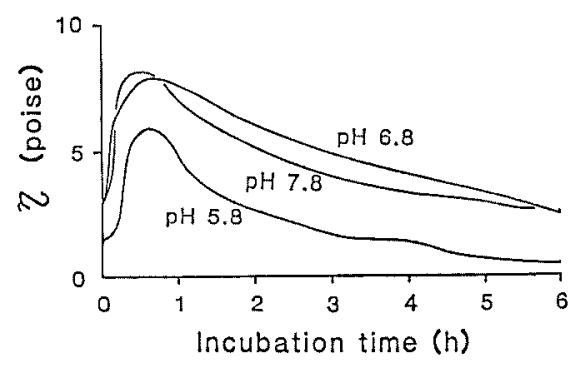

Fig. 1. Effect of the incubation and the repeated mesurements on steady shear rate behavior of carp actomyosin solution.

Actomyosin solution: $19 \mathrm{mg} / \mathrm{ml}$ actomyosin, $0.6 \mathrm{M} \mathrm{NaCl}, 20 \mathrm{~mm}$ tris-maleat buffer.

Incubation temperature $40^{\circ} \mathrm{C}$.

Shear rate $12 \mathrm{~s}^{-1}$.

The viscosity 7 was repeatedly measured at intervals of $10 \mathrm{~min}$ during the incubation (Refer to Materials and Methods). 


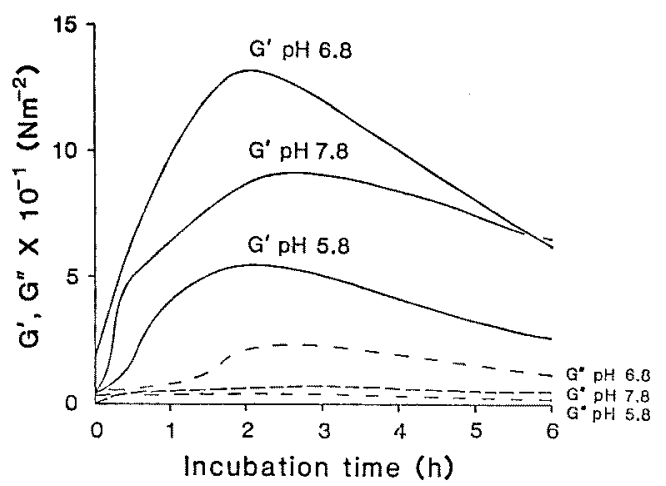

Fig. 2. Effect of the incubation and the repeated measurements on dynamic behavior of carp actomyosin solution.

Actomyosin solution as in Fig. 1.

Incubation temperature $40^{\circ} \mathrm{C}$.

Strain amplitude 0.18 .

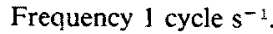

The dynamic rigidity $\left(G^{\prime}\right)$ and the dynamic loss $\left(G^{\prime \prime}\right)$ were repeatedly measured at intervals of 10 min during the incubation (Refer to Materials and Methods).

of dynamic rigidity change during the incubation was similar irrespective of the frequency used. In Fig. 2, the dynamic rigidity measured at 1 cycle $\mathrm{s}^{-1}$, was shown representatively.

The drastic increase of the steady shear viscosity $\eta$ (Fig. 1) and the dynamic rigidity $\mathrm{G}^{\prime}$ (Fig. 2) till $35 \mathrm{~min}$ and $2 \mathrm{~h}$ respectively, indicated the development of the network structure during the incubation. After the incubation time of about $35 \mathrm{~min}, \eta$ began to decrease (Fig. 1). This fact indicated that the network structure was broken down by the large deformation, in the measurement of $\eta$. During the incubation without the measurements of $\eta$ (Fig. 2), the formation process of network structure continued furthermore after $35 \mathrm{~min}$. It was detected by the increase of $G^{\prime}$ till the incubation time of about $2 \mathrm{~h}$ (Fig. 2).

In this place, we explain the result of shear rate scan measurement. Carp actomyosin solutions were incubated at $40^{\circ} \mathrm{C}$ for a given time indicated in Fig. 3. No measurement was made on the way until the indicated time expired.

At the incubation time of $0 \mathrm{~h}$ (before incubation), the relation of shear rate vs. shear stress was represented as an almost straight line passing through the origin of coordinates. The line was slightly curved toward the abscissa near the origin of coordinates. In this experiment, shear rate was once increased and then decreased. The

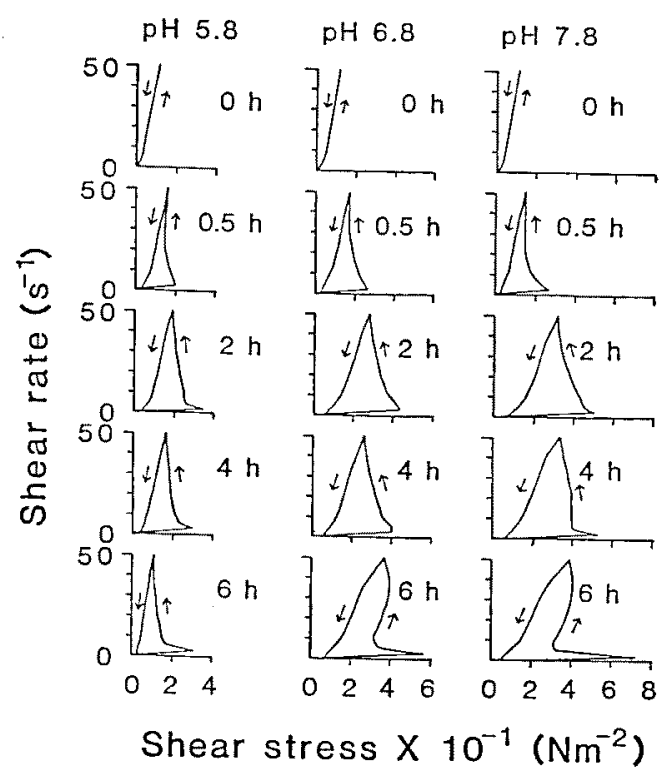

Fig. 3. Effect of the incubation and the single measurement on continuous flow curve of carp actomyosin solution.

Actomyosin solution as in Fig. 1.

Incubation temperature $40^{\circ} \mathrm{C}$.

Shear rate was increased from $1 \mathrm{~s}^{-1}$ to $50 \mathrm{~s}^{-1}$, and then decreased to $1 \mathrm{~s}^{-} \mathbf{t}$ (Refer to Materials and Methods). The continuous flow curve was measured only once for each incubation treatment.

resultant curves were designated as upcurve and downcurve, respectively. In this case the downcurve was retraced almost on the upcurve.

At the incubation time of $0.5 \mathrm{~h}$, the continuous shear curve showed a complex flow behavior. At low values of shear stress the material did not flow. There, the stress reached a critical value corresponding to the peak of the curve. This stress value is designated as yield stress. The yield stress is the minimum shear stress required to produce flow and is considered as a measure of the network structure or solid-like nature. After the yield stress was achieved, the upcurve was non-linear with a negative gradient. This negative, or very steep, gradient in the upcurve is typical of a viscoelastic semisolid ${ }^{8-8)}$ and can be attributed to shear fracture. The downcurve was an almost straight line toward the origin of coordinates, and was displaced to the left of the upcurve indicating a breakdown of structure which did not reform immediately after the stress was diministed. Namely, hysteresis loop was present. 


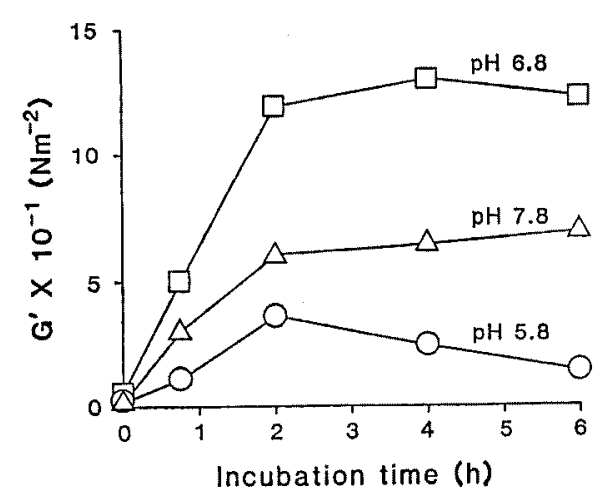

Fig. 4. Effect of the imcubation and the single measurement on dynamic behavior of carp actomyosin solution.

Actomyosin solution as in Fig. 1.

Incubation temperature $40^{\circ} \mathrm{C}$.

Strain amplitude 0.18 .

Frequency 1 cycle $\mathrm{s}^{-} \mathrm{t}$.

The dynamic rigidity $\left(\mathrm{G}^{\prime}\right)$ was measured only once for each incubation treatment (each point of $\square, \triangle$, or $\bigcirc$ in the figure).

From $2 \mathrm{~h}$ till $6 \mathrm{~h}$, the shape of shear rate vs. shear stress curve was similar to that observed at $0.5 \mathrm{~h}$.

At three kinds of $\mathrm{pH}$ (The detailed discussion on the effect of $\mathrm{pH}$ was made in the next chapter.), the hysteresis loop area increased till $2 \mathrm{~h}$, and thereafter it remained almost unchanged till

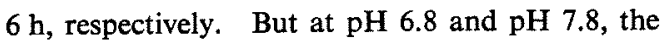
yield stress always continued to increase, even from $2 \mathrm{~h}$ till $6 \mathrm{~h}$, where dynamic rigidity $G^{\prime}$ decreased (Fig. 2). The large yield stresses observed at $6 \mathrm{~h}$ in the environmental condition of $\mathrm{pH} 6.8$ and $\mathrm{pH} 7.8$ suggest the development of the network structure. The structure was broken down during the continuous shear measurement (Fig. 3) to cause the elimination of the increease of the hysteresis loop area, and the structure was not recoverable during the measurement.

As we obtained the above-mentioned results, we reexamined the dynamic shear behavior by the single measurement for each incubation treatment (Fig. 4). As was expected, the dynamic rigidity $\mathrm{G}^{\prime}$ became the maximum at $2 \mathrm{~h}$, and thereafter it remained almost unchanged till $6 \mathrm{~h}$ in the case of $\mathrm{pH} 6.8$ and $\mathrm{pH} 7.8$ (Fig. 4). In the case of $\mathrm{pH}$ $5.8, \mathrm{G}^{\prime}$ became the maximum at $2 \mathrm{~h}$, but thereafter it decreased (Fig. 4). This result obtained at $\mathrm{pH}$ 5.8 (Fig. 4) corresponded to the symptoms observed in the continuous flow curve (Fig. 3). At pH 5.8 in Fig. 3 the hysteresis loop area decreased gradual- ly from $2 \mathrm{~h}$ onward, and the yield stress decreased slightly during this period.

To summarize the results obtained on our carp actomyosin solution, the network structure was broken down also by the small deformation, in the repeated measurements of $G^{\prime}$. The single measurements of both shear rate scan (Fig. 3) and dynamic (Fig. 4) behaviors showed the development of network structure at $\mathrm{pH} 6.8$ and $\mathrm{pH} 7.8$ till $6 \mathrm{~h}$.

These results of the single measurement on carp actomyosin solution were similar to those of the repeated measurements on hen or rabbit myosin solutions. ${ }^{1,2)}$ Therefore we deduce that during the incubation, the network structure world develop also in the carp actomyosin solution as in the hen or rabbit myosin solution, but the network structure of carp actomyosin solution would be so weak as to be broken down even during the repeated measurements of dynamic behaviors. If the viscoelastic change of hen or rabbit actomyosin solution is compared with that of carp actomyosin solution in the furture, this deduction will become clearer.

\section{Effect of $\mathrm{pH}$ on Viscoelastic Properties of Acto- myosin Solution}

The effect of $\mathrm{pH}$ on the charge state of actomyosin is important because the change of $\mathrm{pH}$ induces the formation or breakdown of the interprotein ionic linkages and the shrinkage or swelling of the interprotein network structure. From this viewpoint, the $\mathrm{pH}$ dependence of the viscoelastic properties of actomyosin solution was investigated during the incubation at $40^{\circ} \mathrm{C}$.

There was not the obvious difference of the steady shear viscosity between the actomyosin solutions at $\mathrm{pH} 6.8$ and $\mathrm{pH} 7.8$ when compared at any incubation time (Fig. 1). The actomyosin solution at $\mathrm{pH} 5.8$ was the lowest in the steady shear viscosity along the course of the incubation.

The dynamic rigidity of actomyosin solution (Figs. 2 and 4) was high at $\mathrm{pH} \mathrm{6.8,} \mathrm{medium} \mathrm{at}$ pH 7.8, and low at pH 5.8.

In the shear rate scan measurement (Fig. 4), the $\mathrm{pH}$ dependent difference of the shear stress was not found on the incubation time of $0 \mathrm{~h}$ (before incubation). On the incubation time of $0.5 \mathrm{~h}$, both the shear stress and the hysteresis loop area were larger at $\mathrm{pH} 6.8$ and $\mathrm{pH} 7.8$ than at $\mathrm{pH}$ 5.8. On the incubation time of $2 \mathrm{~h}$, both the hysteresis loop area and the yield stress were the largest at pH 7.8 and the smallest at $\mathrm{pH} 5.8$. 
On the incubation times of $4 \mathrm{~h}$ and $6 \mathrm{~h}$, the yield stress was the largest at $\mathrm{pH} 7.8$ and the smallest at $\mathrm{pH} 5.8$.

To summarize our results, the actomyosin solution at pH 5.8 exhibited the lowest viscoelasticity (Figs. 1, 2, 3, and 4). The actomyosin solution at pH 6.8 exhibited the highest dynamic righidity (Figs. 2 and 4). The actomyosin solution at $\mathrm{pH} 7.8$ exhibited the highest stress in the shear rate scan measurement (Fig. 3).

The isoelectric point of actomysoin is located between $\mathrm{pH} 5.0$ and $\mathrm{pH} 5.5 .^{\circ)}$ Our actomyosin solution at $\mathrm{pH} 5.8$ existed near the isoelectric point. Whitaker and Tannenbaum ${ }^{10)}$ described that on the basic side of the isoelectric point, negative charges will prevail and the protein molecules will tend to repel each other, the matrix will swell, and water holding capacity will increase. Hamm $^{11)}$ described that the higher the swelling and the water holding capacity of minced meat, the greater the shearing stress required to make it flow. He reported that near the isoelectric point (pH 5.5), the shear and yield stresses of minced meat were lower. His results coincided with ours.

The discrepancy of the order in the magnitude between the dynamic rigidity ( $\mathrm{G}^{\prime}$ ) (Figs. 2 and 4) and the hysteresis loop area/the yield stress (Fig. 3) was observed on $\mathrm{pH} 6.8$ and $\mathrm{pH}$ 7.8. This would be probably due to the difference of network structure. It was deduced that the network structure at pH 6.8 might be fine and might contain more entaglements, on the assumption that fine network structure shows high dynamic rigidity and the entanglements get untied by much lower stresses than the cross-linke are broken down. On the other hand, it was suggested for the same reasons that the network structure at $\mathrm{pH} 7.8$ might be coarse and might contain more cross-links. Therefore in the small deformation (Figs. 2 and 4) the higher dynamic rigidity was observed at $\mathrm{pH}$ 6.8 , and in the large deformation (Fig. 3) the higher shear stress was required at $\mathrm{pH} 7.8$.

\section{Rigidity Change through Temperature Scanning}

In this experiment, we used actomyosin solution of rather lower concentration $16 \mathrm{mg} / \mathrm{m} l$ because the upper limit of the rheometer was $140 \mathrm{Nm}^{-2}$ in the dynamic rigidity $\mathrm{G}^{\prime}$.

As presented in Fig. 5, the obvious increase of dynamic rigidity was detected around $40^{\circ} \mathrm{C}$, and the increase became much more conspicuous beyond $45^{\circ} \mathrm{C}$. This steep increase reached maximum around $61^{\circ} \mathrm{C}$, and the dynamic rigidity be-

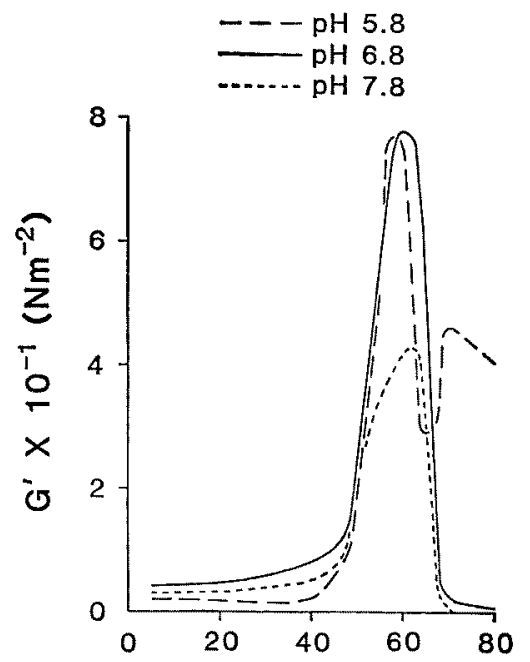

Temp. of actomyosin solution $\left({ }^{\circ} \mathrm{C}\right)$

Fig. 5. Effect of temperature on dynamic behavior of carp actomyosin solution.

Actomyosin solution: $16 \mathrm{mg} / \mathrm{ml}$ actomyosin, $0.6 \mathrm{M} \mathrm{NaCl}, 20 \mathrm{~mm}$ tris-maleate buffer.

Strain amplitude 0.18 .

Frequency 1 cycle s $^{-1}$.

The dynamic rigidity $\left(\mathrm{G}^{\prime}\right)$ was repeatedly measured at intervals of 5 or $3^{\circ} \mathrm{C}$ during the temperature scanning.

gan to decrease very steeply beyond this temperature range. The dynamic rigidity almost disappeared around $71^{\circ} \mathrm{C}$ in the case of $\mathrm{pH} 6.8$ and $\mathrm{pH}$ 7.8. The tendency of the temperature dependent change of the dynamic rigidity was similar irrespective of the difference of $\mathrm{pH}$. But the peak value around $61^{\circ} \mathrm{C}$ was hihg at $\mathrm{pH} 5.8$ and $\mathrm{pH}$ 6.8, and low at $\mathrm{pH} 7.8$.

Around $40^{\circ} \mathrm{C}$, the dynamic rigidity $\mathrm{G}^{\prime}$ was the lowest at pH 5.8 (Fig. 5) and this tendency was the same as that shown in Figs. 2 and 4 . This result would be due to the coarse network structure (i.e. less entanglements and less cross-links). According to Whitaker and Tannenbaum, ${ }^{10)}$ the water holidng capacity is the lowest at this $\mathrm{pH}$ range near the isoelectric point. When the temperture was elevated from $40^{\circ} \mathrm{C}$ to $61^{\circ} \mathrm{C}$ at $\mathrm{pH} 5.8$, some amount of the loosely entrapped water within the protein network would be separated to lead the approach of the neighbouring protein chains the coagulation. The high dynamic rigidity around $61^{\circ} \mathrm{C}$ at pH 5.8 (Fig. 5) would be caused through this process.

Around $40^{\circ} \mathrm{C}, \mathrm{G}^{\prime}$ at $\mathrm{pH} 6.8$ was higher than $\mathrm{G}^{\prime}$ $\mathrm{G}^{\prime}$ at pH 7.8 (Fig. 5), and this tendency was the 
same as that shown in Figs. 2 and 4. The reason of this result was discussed in the preceding chapter and we considered that the netwoork structure was finer at $\mathrm{pH} 6.8$ than at $\mathrm{pH} 7.8$. When the temperature was elevated from $40^{\circ} \mathrm{C}$ to $61^{\circ} \mathrm{C} \mathrm{G}^{\prime}$ at $\mathrm{pH} 6.8$ was also higher than $\mathrm{G}^{\prime}$ at $\mathrm{pH} 7.8$ and this tendency became more remarkable (Fig. 5). This result suggests that the above-mentioned network structures at $\mathrm{pH} 6.8$ and $\mathrm{pH} 7.8$ were reinforced during heating up to $61^{\circ} \mathrm{C}$, in proportion to their early structures around $40^{\circ} \mathrm{C}$.

The $\mathrm{pH}$ dependency of the peak rigidity around $61^{\circ} \mathrm{C}$ found in our carp actomyosin solution (Fig. 5 ), was similar to the result obtained on rabbit myosin solution by Ishioroshi et al. ${ }^{12)}$ In other words, the viscoelastic properties of actomyosin solution might be revealed by the properties of its component myosin. This assumption will be proved when carp myosin solution is investigated in the future.

The shape of the rigidity vs. temperature curve obtained on our carp actomyosin solution (Fig. 5 ), was similar to the shape of the turbidity vs. temperature curve obtained on hen actomyosin solution (pH 7.0) by Nakayama and Sato. ${ }^{13)}$ In other words, heat denaturation of actomyosin was a common nature between fish and domestic animal.

Beyond the peak around $61^{\circ} \mathrm{C}$, the rigidity was kept almost constant in Ishioroshi's result, ${ }^{12)}$ but the rigidity $\left(\mathrm{G}^{\prime}\right)$ decreased in our result (Fig. 5). In this respect, we conclude that the network structure of carp actomyosin solution is more easily broken by the high temperature and the repeated measurements, as compared to the network structure of rabbit myosin solution.

In the case of $\mathrm{pH} 5.8$, beyond the peak around $61^{\circ} \mathrm{C}$, the dynamic rigidity once decreased, then increased till $66^{\circ} \mathrm{C}$, and thereafter decreased again. This result was reliable, reproducible, and quite different from the result obtained at $\mathrm{pH}$ 6.8 and $\mathrm{pH}$ 7.8. It is undoubted that beyond the peak around $61^{\circ} \mathrm{C}$, irrespective of $\mathrm{pH}$, the transition of the network structure occurred, and the peculiar behavior at pH 5.8 was ascribed to the state in the vicinity of the isoelectric point. The meaning of these results is left to be elucidated from the physicochemical aspects.

\section{References}

1) T. Nakayama, M. Shirai, N. Kanome, E. Niwa, and I. Hamada: Nippon Suisan Gakkaishi, 41, 1283-1286 (1975).

2) T. Nakayama, H. Okuda, E. Niwa, and I. Hamada Nippon Suisan Gakkaishi, 40, 937-940 (1974).

3) T. Nakayama, E. Niwa, I. Hamada, and C. Shin: J. Food Sci., 44, 1106-1109 (1979).

4) R. Takashi, K. Arai, and T. Saito: Nippon Suisan Gakkaishi, 36, 165-168 (1970).

5) T. Nakayama, M. Kawasaki, E. Niwa, and I. Hamada: J. Food Sci., 43, 1430-1432 (1978).

6) T. Nakayama and P. Haugen: J. Texture Studies, 8, 81-91 (1977).

7) S.S. Davis, M.S. Khanderia, I. Adams, I. R. Colley, J. Cammack, and P. Sanford: J. Texture Studiues, 8, 61-80 (1977).

8) M. A. Bianchi, A. M. R. Piısof, and G. B. Bartholomai: J. Texture Studies, 16, 193-206 (1985).

9) H. R. Mahler and E. H. Cordes: Biological Chemistry, Harper International Editions, New York, 1966, pp. 54.

10) Food Proteins, (ed, by J. R. Whitaker and S. R. Tannenbaum), AVI Publishing Co., Inc., Westport, 1977, pp. 78-85.

11) R. Hamm: J. Texture Studies, 6, 281-296 (1975).

12) M. Ishiorcshi, K. Samejima, and T. Yasui: $J$. Food Sci., 44, 12ع0-1284 (1979).

13) T. Nakayama and Y. Sato: J. Texture Studies, 2, 475-488 (1971). 